\title{
WAVE NUMBER OF MAXIMAL GROWTH IN VISCOUS FERROFLUIDS
}

\author{
A. Lange ${ }^{1}$, B. Reimann ${ }^{2}$, R. Richter ${ }^{2}$ \\ ${ }^{1}$ Universität Magdeburg, Institut für Theoretische Physik, Postfach 4120, \\ D-39106 Magdeburg, Germany \\ ${ }^{2}$ Universität Bayreuth, Physikalisches Institut, Experimentalphysik $V$, \\ D-95440 Bayreuth, Germany
}

\begin{abstract}
Within the frame of linear stability theory an analytical method is presented for the normal field instability in magnetic fluids. It allows to calculate the maximal growth rate and the corresponding wave number for arbitrary values of the layer thickness and viscosity. Applying this method to magnetic fluids of finite depth, the results are quantitatively compared to the wave number of the transient pattern observed experimentally after a jumplike increase of the field. The wave number grows linearly with increasing induction where the theoretical and the experimental data agree well.
\end{abstract}

Introduction. The most striking phenomenon of pattern formation in magnetic fluids is the Rosensweig or normal field instability [1]-[4]. Above a threshold $B_{c}$ of the induction, the initially flat surface exhibits a stationary hexagonal pattern of peaks. Typically, patterns are characterized by a wave vector $\mathbf{q}$ whose absolute value gives the wave number $q=|\mathbf{q}|$. There are few but contradictory experimental observations. In experiments where the field is increased continuously, there are reports about constant $[1,5]$ as well as about varying wave numbers [6]. Notably, all these observations are of entirely qualitative character.

All qualitative observations refer to the final arrangement of peaks. The final stable pattern, resulting from nonlinear interactions, does not generally correspond to the most unstable linear pattern. Such a pattern should grow with the maximal growth rate and should display the corresponding wave number. Since both quantities are calculated by the linear theory, the most unstable linear pattern has to be detected and measured experimentally for a meaningful comparison between theory and experiment.

1. Theory. A horizontally unbounded layer of an incompressible, nonconducting, and viscous magnetic fluid of thickness $h$ and constant density $\rho$ is considered. The fluid is bounded from below by the bottom of a container made of a magnetically impermeable material and has a free surface with air above. The electrically insulating fluid justifies the stationary form of the Maxwell equations, which reduce to the Laplace equation for the magnetic potentials in each of the three different regions. It is assumed that the magnetization of the magnetic fluid depends linearly on the applied magnetic field, $\mathbf{M}=\left(\mu_{r}-1\right) \mathbf{H}$, where $\mu_{r}$ is the relative permeability of the fluid.

In a linear stability analysis, all small disturbances from the basic state are analysed into normal modes, i.e., they are proportional to $\exp [-i(\omega t-\mathbf{q} \mathbf{r})]$. If $\operatorname{Im}(\omega)>0$, initially small undulations will grow exponentially and the originally horizontal surface is unstable. Therefore it has been established to denote $\omega$ as growth rate, which is in fact true only for its imaginary part in the chosen normal mode ansatz. Following the standard procedure, the linear stability analysis leads 
to the dispersion relation [7]-[9] (all formulas in the references are equivalent to each other)

$$
\begin{aligned}
& \frac{\nu^{2}}{\tilde{q} \operatorname{coth}(\tilde{q} h)-q \operatorname{coth}(q h)}\left\{\tilde{q}\left[4 q^{4}+\left(q^{2}+\tilde{q}^{2}\right)^{2}\right] \operatorname{coth}(\tilde{q} h)\right. \\
& \left.-q\left[4 q^{2} \tilde{q}^{2}+\left(q^{2}+\tilde{q}^{2}\right)^{2}\right] \tanh (q h)-\frac{4 q^{2} \tilde{q}\left(q^{2}+\tilde{q}^{2}\right)}{\cosh (q h) \sinh (\tilde{q} h)}\right\} \\
& +\tanh (q h)\left[g q+\frac{\sigma}{\rho} q^{3}-\frac{\mu_{0} \mu_{r} M^{2}}{\rho} \Lambda(q h) q^{2}\right]=0,
\end{aligned}
$$

where $\mu_{0}$ is the permeability of free space, $\nu$ the kinematic viscosity, $\tilde{q}=\sqrt{q^{2}-i \omega / \nu}$, and

$$
\Lambda(q h)=\frac{\mathrm{e}^{q h}\left(1+\mu_{r}\right)+\mathrm{e}^{-q h}\left(1-\mu_{r}\right)}{\mathrm{e}^{q h}\left(1+\mu_{r}\right)^{2}-\mathrm{e}^{-q h}\left(1-\mu_{r}\right)^{2}} .
$$

The condition of marginal stability, $\omega=0$, defines the threshold where $\omega$ changes its sign and therefore the normal field or Rosensweig instability appears. With $\omega=0$, Eq. (1) reduces to

$$
\frac{\rho g}{\sigma}+q^{2}-\frac{\left(\mu_{r}-1\right)^{2} B^{2}}{\mu_{0} \mu_{r} \sigma} q \Lambda(q h)=0 .
$$

In the limit of an infinitely thick layer $(h \rightarrow \infty)$, the critical induction and the critical wave number are [1]

$$
B_{c, \infty}^{2}=\frac{2 \mu_{0} \mu_{r}\left(\mu_{r}+1\right) \sqrt{\rho \sigma g}}{\left(\mu_{r}-1\right)^{2}} \quad q_{c, \infty}=\sqrt{\frac{\rho g}{\sigma}} .
$$

These critical values apply to both viscous and inviscid magnetic fluids due to the static character of the instability.

1.1. Infinite layer. The starting point of the analysis is the determination of the parameters for which the dispersion relation (1) for an infinitely thick layer [10]

$$
\left(1-\frac{i \omega}{2 \nu q^{2}}\right)^{2}+\frac{1}{4 \rho \nu^{2} q^{4}}\left[\rho g q+\sigma q^{3}-\frac{\left(\mu_{r}-1\right)^{2}}{\left(\mu_{r}+1\right) \mu_{0} \mu_{r}} B^{2} q^{2}\right]=\sqrt{1-\frac{i \omega}{\nu q^{2}}}
$$

has solutions of purely imaginary growth rates. Dimensionless quantities are introduced for all lengths, the induction, the time, and the viscosity

$$
\begin{aligned}
\bar{l} & =q_{c, \infty} l, & \bar{B} & =\frac{B}{B_{c, \infty}}, \\
\bar{t} & =\frac{g^{3 / 4} \rho^{1 / 4}}{\sigma^{1 / 4}} t=\frac{t}{t_{c}}, & \bar{\nu} & =\frac{g^{1 / 4} \rho^{3 / 4}}{\sigma^{3 / 4}} \nu,
\end{aligned}
$$

where $t_{c}$ is the so-called capillary time. The real part of Eq. (5) reduces for $\bar{\omega}=i \bar{\omega}_{2}$ to

$$
f_{ \pm}(\bar{q},|\bar{\omega}| ; \bar{\nu}, \bar{B}):=\left(\bar{\nu} \pm \frac{|\bar{\omega}|}{2 \bar{q}^{2}}\right)^{2}+\frac{\bar{q}+\bar{q}^{3}-2 \bar{B}^{2} \bar{q}^{2}}{4 \bar{q}^{4}}-\bar{\nu}^{2} \sqrt[4]{\left(1 \pm \frac{|\bar{\omega}|}{\bar{\nu} \bar{q}^{2}}\right)^{2}}=0
$$

where the \pm sign corresponds to $\bar{\omega}_{2} \gtrless 0$. The parameters $\bar{\nu}$ and $\bar{B}$ determine the solution of this implicit equation for the variables $\bar{q}$ and $|\bar{\omega}|$. For supercritical inductions, the solutions of Eq. (8) have a maximum in the growth rate $\bar{\omega}_{m}=i \bar{\omega}_{2, m}$ 


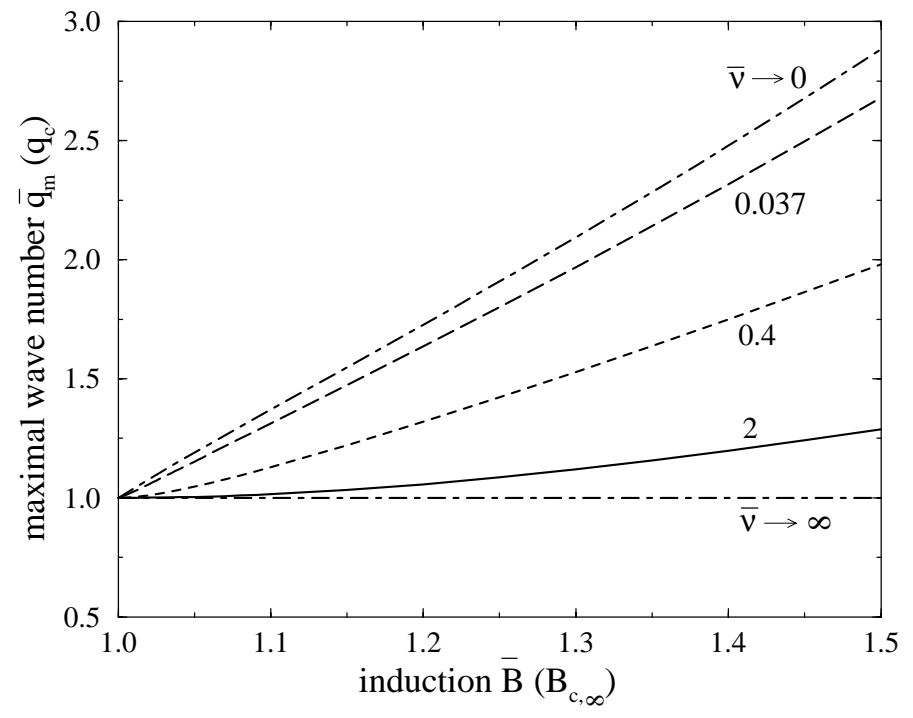

Fig. 1. Maximal wave number $\bar{q}_{m}$ as a function of the supercritical induction $\bar{B}$ for different viscosities. $\bar{q}_{m}$ is a monotonously increasing function of $\bar{B}$ with the exception $\bar{q}_{m}=1[10]$ in the case of infinitely large viscosities (lower dot-dashed line). In the limit of an inviscid fluid (upper dot-dashed line) the dependence of $\bar{q}_{m}$ on $\bar{B}$ is given by $\bar{q}_{m}=(1 / 3)\left(2 \bar{B}^{2}+\sqrt{4 \bar{B}^{4}-3}\right)[7]$.

at $\bar{q}_{m}$. The wave number with the maximal growth rate is defined by $\partial \bar{\omega}_{2} / \partial \bar{q}=$ $\partial|\bar{\omega}| / \partial \bar{q}=0$. Since $|\bar{\omega}|$ is given implicitly by $f_{ \pm}(\bar{q},|\bar{\omega}| ; \bar{\nu}, \bar{B})=0$, the maximal growth rate results from $\partial_{\bar{q}} f_{+}=0$. This condition gives a second implicit function whose specific form is given in [11]. The cross section of the solutions of $f_{+}=0$ and $\partial_{\bar{q}} f_{+}=0$ gives $\left|\bar{\omega}_{m}\right|$ and $\bar{q}_{m}$, which is shown for different viscosities in Fig. 1.

For all finite viscosities, the wave number $\bar{q}_{m}$ is not constant, i.e., for finite viscosities $\bar{q}_{m}$ depends on the external control parameter $\bar{B}$. With increasing viscosity $\bar{q}_{m}$ varies less with increasing induction. For small viscosities $\bar{q}_{m}$ depends linearly on $\bar{B}$ if $\bar{B}$ is not too large. The analysis reveals that only in the case of infinitely large viscosities a constant wave vector of maximal growth $\bar{q}_{m}=1$ can be expected. The experimental observation in $[1,5]$ cannot be explained by the result of an asymptotic analysis [10] in which the case of an infinitely viscous fluid was considered. Experimental fluids $[6,12]$ show a viscosity of $\bar{\nu}=0.037\left(\nu \simeq 6.4 \times 10^{-6} \mathrm{~m}^{2} \mathrm{~s}^{-1}\right)$, i.e., realistic magnetic fluids tend rather to the limit $\bar{\nu} \rightarrow 0$.

1.2. Finite layer. Since the experiments are performed with a vessel of finite depth, the analysis presented in the preceding section has to be applied to magnetic fluids of finite thickness. The implicit equation

$$
\begin{aligned}
& f_{+}(q, \omega ; \nu, B, h):=\frac{\nu^{2}\left[\cosh \left(2 \tilde{q}_{1} h\right)-1\right]}{\tilde{q}_{1} \sinh \left(2 \tilde{q}_{1} h\right)-q \operatorname{coth}(q h)\left[\cosh \left(2 \tilde{q}_{1} h\right)-1\right]} \times \\
& \left\{\frac{\tilde{q}_{1} \sinh \left(2 \tilde{q}_{1} h\right)\left(5 q^{4}+2 q^{2} \tilde{q}_{1}^{2}+\tilde{q}_{1}^{4}\right)}{\cosh \left(2 \tilde{q}_{1} h\right)-1}-q \tanh (q h)\left(5 q^{2} \tilde{q}_{1}^{2}+q^{4}+\tilde{q}_{1}^{4}\right)\right. \\
& \left.-\frac{4 q^{2} \tilde{q}_{1}\left(q^{2}+\tilde{q}_{1}^{2}\right)}{\cosh (q h) \sinh \left(\tilde{q}_{1} h\right)}\right\}+\tanh (q h)\left[g q+\frac{\sigma}{\rho} q^{3}-\frac{\left(\mu_{r}-1\right)^{2} B^{2}}{\mu_{0} \mu_{r} \rho} \Lambda(q h) q^{2}\right]=0,
\end{aligned}
$$



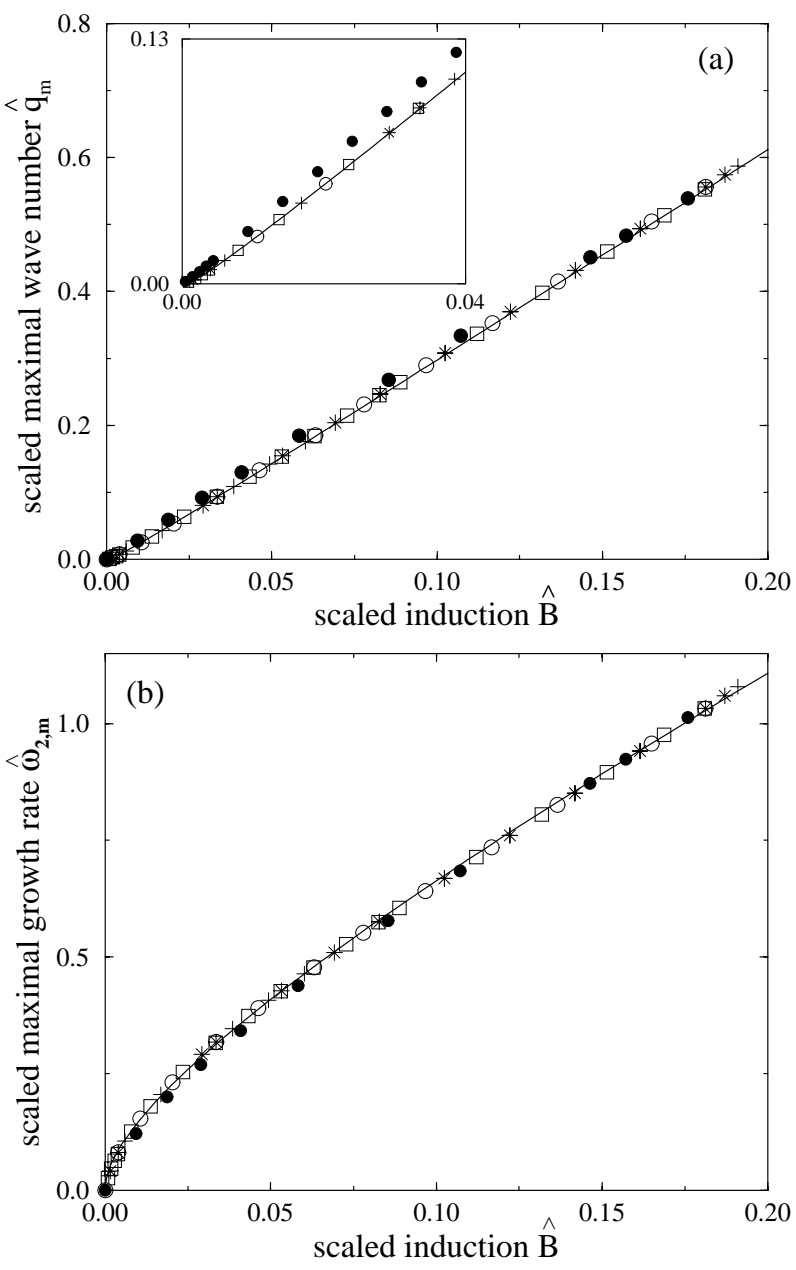

Fig. 2. Scaled maximal wave number $\hat{q}_{m}$ (a) and scaled maximal growth rate $\hat{\omega}_{2, m}$ (b) as a function of the scaled supercritical induction $\hat{B}$. The data are calculated for $h=100$ $\mathrm{mm}(\circ), 50 \mathrm{~mm}(*), 10 \mathrm{~mm}(+), 4 \mathrm{~mm}(\square), 2 \mathrm{~mm}(\bullet)$. For $h \geq 4 \mathrm{~mm}$, the data are fitted by $\hat{q}_{m}=3.32 \hat{B}-0.10 \sqrt{\hat{B}}$ for $\hat{q}_{m}$ [solid line (a)] and by $\hat{\omega}_{2, m}=1.16 \sqrt{\hat{B}}+2.95 \hat{B}$ for $\hat{\omega}_{2, m}$ [solid line (b)]. Small deviations from the generic behaviour can be seen for $h=2 \mathrm{~mm}$ (inset). Material parameters of EMG 901: $\mu_{r}=4.0, \rho=1.53 \cdot 10^{3} \mathrm{~kg} \cdot \mathrm{m}^{-3}$, $\nu=6.54 \cdot 10^{-6} \mathrm{~m}^{2} \cdot \mathrm{s}^{-1}$, and $\sigma=2.27 \cdot 10^{-2} \mathrm{~kg} \cdot \mathrm{s}^{-2}$.

for the variables $q$ and $\omega$ contains now the additional parameter $h$. The influence of the layer thickness $h$ on the solution were studied in detail in [11]. This study displayed that the wave number of maximal growth $q_{m}$ is less sensitive to changes in the layer thickness than the maximal growth rate $\omega_{2, m}$.

To analyse the behaviour of $\omega_{2, m}$ and $q_{m}$ on $B$ and $h$, again the cross section of the solutions of $f_{+}=0$ and $\partial_{q} f_{+}=0$ has to be determined. Through the implicit character of the functions, a general analytical expression cannot be given for the dependence of $q_{m}$ and $\omega_{2, m}$ on $\nu, B$ and $h$. Alternatively, a two-parameter fit is tested, which describes the generic behaviour of $q_{m}$ and $\omega_{2, m}$ on $B$ and $h$ over a wide range of layer thicknesses. An excellent agreement is achieved for $h \geq 4 \mathrm{~mm}$ 
by

$$
\begin{aligned}
\hat{q}_{m} & =3.32 \hat{B}-0.10 \sqrt{\hat{B}} & & \text { for } 0.005 \leq \hat{B} \leq 0.4 \\
\hat{\omega}_{2, m} & =1.16 \sqrt{\hat{B}}+2.95 \hat{B} & & \text { for } 0.005 \leq \hat{B} \leq 0.4
\end{aligned}
$$

(see Fig. 2), where $\hat{B}=\left(B-B_{c, h}\right) / B_{c, h}$ and $\hat{q}_{m}=\left(q_{m}-q_{c, h}\right) / q_{c, h}$ denote the scaled distances from the critical values. The dimensionless growth rate is given by $\hat{\omega}_{2, m}=\omega_{2, m} t_{c}$. For small $\hat{B}$, the behaviour of $\hat{q}_{m}$ is only weakly nonlinear whereas the behaviour of $\hat{\omega}_{2, m}$ is determined by the square-root term. A careful inspection of the data reveals that for $h=2 \mathrm{~mm}$ (filled circles), small deviations from the proposed fits appear: $\hat{q}_{m}$ grows linearly over the entire $\hat{B}$ region [see inset in Fig. 2 (a)]. Thus $h=2 \mathrm{~mm}$ indicates the lower limit of the validity of $(4,5)$ with respect to the layer thickness.

2. Experiment and Comparison with Theory. Let us start with a sketch of the experimental setup. A more detailed description can be found in [11]. We place a cylindrical Teflon ${ }^{\circledR}$ vessel with a diameter of $\mathrm{d}=12 \mathrm{~cm}$ and a depth of $2 \mathrm{~mm}$, completely filled with magnetic fluid (EMG 909), in the center of a pair of Helmholtz coils. A CCD-camera is positioned above the vessel in the center of a ring of LEDs. By this construction only an inclined surface of proper angle will reflect light into the camera. In the theoretical analysis the supercritical magnetic field is assumed to be instantly present, thus in the experiment the magnetic field has to be increased jump-like from a subcritical value $B_{0}$ to the desired value $B$. For all measurements $B_{0}$ was fixed to $133 \cdot 10^{-4} \mathrm{~T}$. The induction is recorded by a Siemens Hall-probe (KSY 13) with short relaxation time, positioned immediately under the vessel.

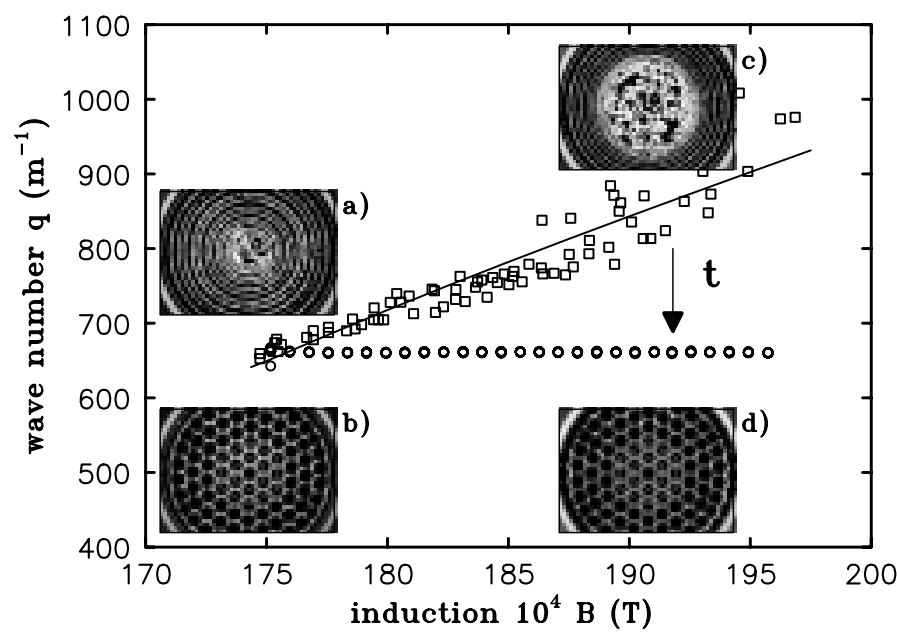

Fig. 3. Plot of the wave number $q$ versus the magnetic induction $B$. The open squares give the experimental values extracted from the circular deformations, examples of which are given in the insets a), c). The solid line displays the theoretical results for the material parameters of EMG 909: $\rho=1.53 \cdot 10^{3} \mathrm{~kg} \cdot \mathrm{m}^{-3}, \nu=6.5410^{-6} \mathrm{~m}^{2} \cdot \mathrm{s}^{-1}$, and $\sigma=2.27 \cdot 10^{-2} \mathrm{~kg} \cdot \mathrm{s}^{-2}$, using $\mu_{r} \simeq 1.85$ as a fit-parameter. The open circles denote the wave number of the final hexagonal patterns (see inset b), c) e.g.) calculated via a fit of the central hexagonal structure. 
Fig. 3a) shows circular surface deformations taken $180 \mathrm{~ms}$ after a jump like increase of the induction. After this transient concentric arrangement a hexagonal pattern of Rosensweig peaks evolves (see Fig. $3 \mathrm{~b}$ )). The evolution of the pattern can be understood as a transition from a one dimensional unstable solution [13] to a stable hexagonal one (see Fig. 2 in [3]).

Next we focus on the experimental results displayed in Fig. 3, where the wave number $q$ is plotted versus the magnetic induction $B$. Each open square denotes the wave number extracted from a picture taken during a jump-like increase of the magnetic field to $B>B_{c}$. The estimated maximal errors for $q$ of $\pm 4.2 \%$ and for $B$ of $\pm 0.9 \%$ are not plotted for the purpose of clarity. Using $\mu_{r}$ as a fit-parameter gives the solid line with $\mu_{r} \simeq 1.85$. The fitted value for $\mu_{r}$ differs by $2.8 \%$ from the value given by Ferrofluidics, a deviation which is well within the tolerance of production specified by Ferrofluidics. Obviously there is a rather good agreement between the experimental results and the theoretical graph. In contrast to this linear dependence we find a constant behaviour for the wave number of the final hexagonal pattern, which is marked by the open circles. This measured constant value confirms the qualitative observations for the final pattern in $[1,5]$. The experimental data in Fig. 3 show convincingly the difference between the linear and nonlinear stages of the pattern forming process.

To conclude, we have presented an analytical method which allows to calculate the wave number of maximal growth for any combination of experimental parameters. It has been applied to a liquid layer of $2 \mathrm{~mm}$ thickness. We have demonstrated that the transient pattern is the most suitable one to be compared to the linear theory. The linear increase in the appearing wave number, both in experiment and in theory, is our main outcome. The induction independent behaviour of the final wave number is in agreement with previous observations. However, it is correlated with a nonlinear state which should not be compared with a linear theory.

Acknowledgments. The authors profit from stimulating discussions with J. Berg, A. Engel, R. Friedrichs, H. W. Müller, I. Rehberg and A. Rothert. This work was supported by the Deutsche Forschungsgemeinschaft under Grant EN 278/2 and LA 1182/2-1.

\section{REFERENCES}

1. M. D. Cowley and R. E. Rosensweig. The interfacial stability of a ferromagnetic fluid. J. Fluid Mech., vol. 30 (1967), no. 4, pp. 671-688.

2. R. E. Rosensweig. Ferrohydrodynamics (Cambridge University Press, Cambridge, 1985).

3. A. Gailitis. Formation of the hexagonal pattern of the surface of a ferromagnetic fluid in an applied magnetic field. J. Fluid Mech., vol. 82 (1977), no. 3, pp. 401-413.

4. V. G. Bashtovoi, M. S. Krakov, and A. G. Recks. Instability of a flat layer of magnetic liquid for supercritical magnetic fields. Magnetohydrodynamics, vol. 21 (1985), no. 1, pp. 14-19.

5. J.-C. BaCRi AND D. SAlin. First-order transition in the instability of a magnetic fluid interface. J. Phys. (France), vol. 45 (1984), no. 11, pp. L559-L564.

6. T. MAнr. Oberflächeninstabilitäten bei Ferrofluiden. Ph.D. thesis, Universität Magdeburg, 1998.

7. B. Abou, G. Néron de Surgy, and J. E. Wesfreid. Dispersion relation in a ferrofluid layer of any thickness and viscosity in a normal magnetic field; asymptotic regimes. J. Phys. II, vol. 7 (1997), no. 8, pp. 1159-1171. 
8. J. Weilepp And H. R. BRand. Competition between the Bénard-Marangoni and the Rosensweig instability in magnetic fluids. J. Phys. II, vol. 6 (1996), no. 3, pp. 419441.

9. H. W. MüLlER. Parametrically driven surface waves on viscous ferrofluids. Phys. Rev. E, vol. 58 (1998), no. 5, pp. 6199-6205.

10. D. Salin. Wave vector selection in the instability of an interface in a magnetic or electric field. Europhys. Lett., vol. 21 (1993), no. 6, pp. 667-670.

11. A. Lange, B. Reimann, And R. Richter. Wave number of maximal growth in viscous magnetic fluids of arbitrary depth. Phys. Rev. E, vol. 61 (2000), no. 5, pp. 5528-5539.

12. T. Mahr and I. Rehberg. Nonlinear dynamics of a single ferrofluid-peak in an oscillating magnetic field. Physica D, vol. 111 (1998), pp. 335-346.

13. V. M. Zaitsev, And M. I. Shliomis. Doklady Akad. Nauk S.S.S.R., vol. 188 (1969), pp. 1261-1264; english translation: V.M. ZaitZev And M.I. Shliomis. Nature of the instability of the interface between two liquids in a constant field. Sov. Phys.Doklady, vol. 14 (1970), no. 10, pp. 1001-1002.

Received 22.05.2001 